\title{
Impact of dose rounding of cancer therapy on cost avoidance: a pilot study
}

Nagwa Ibrahim ${ }^{1}$

Department of Pharmaceutical Services, Prince Sultan Military Medical City, Saudi Arabia

\begin{abstract}
BACKGROUND: A significant and progressive cost rising in medical oncology due to the incorporation of novel and highly expensive drugs into clinical practice have been seen in the past ten years. Dose rounding is an option might be used in oncology settings to avoid extra cost. The purpose of this project is to determine the theoretical cost saving related to a dose rounding process for biological and chemotherapy agents in adult oncology settings and to determine the opinion of oncologists about dose rounding.

MATERIALS AND METHODS: Data was obtained prospectively during April 2011. All chemotherapy and targeted therapy orders prescribed in adult oncology outpatient clinics as well as in-patient wards have been collected. We considered rounding to an amount within $15 \%$ for targeted therapy and $10 \%$ for cytotoxic drugs. Chemotherapy dosing was calculated according to body surface area. Prescriptions that include cancer therapy in doses that might be rounded according to study criteria were identified.

RESULTS: Two hundred and thirty three orders of chemotherapy and targeted therapy were processed by Adult Oncology Satellite Pharmacy during the period of data collection. Forty percent of the collected prescriptions fulfilled the criteria. The potential cost savings from dose rounding per year was $\$ 192,800$. Data was extrapolated from the determined monthly cost savings. The highest cost saving was for breast cancer orders $\$ 80,820(42 \%)$, followed by colorectal cancer $\$ 47,965(25 \%)$, while in non-Hodgkin's lymphoma cost savings was \$ 45,107 (23\%) and for other types of cancer that include non small cell lung cancer, prostate and ovarian cancer, in addition to head and neck cost savings was $\$ 18,867(10 \%)$.

CONCLUSIONS: Our experience confirms the significant cost savings of cancer therapy by applying dose rounding to chemotherapy and biologic drugs prescriptions. While clinical impact of the suggested percentage needs to be evaluated.
\end{abstract}

\section{Keywords}

Cancer therapy, Dose rounding, Cost-saving

\section{BACKGROUND}

Cancer is one of the top leading causes of death worldwide. Its prevalence is increasing yearly. This led to advancement in oncology research and development of competitive oncology drugs to help cancer patients and improve clinical therapeutic outcomes. Cancer costs were estimated to reach 157 billion dollars by 2020 , representing a $600 \%$ increase over the past $[1,2]$.

The incorporation of highly expensive novel drugs such as biologic anticancer agents and oral chemotherapy into clinical practice leads to significant and progressive cost rising in medical oncology to unexpected limit. Dose rounding of cancer therapy was considered in few studies conducted by oncologists and clinical pharmacists involved in cancer management. In these literatures dose rounding of both chemotherapy and biologic agents was applied either to the nearest vial size or up/ down within a defined range. Authors reported the results with significant cost saving [27]. The most common method of calculating the dose of anticancer drugs is body surface area (BSA); a function of a patient's height and weight in addition to per weight basis. There is a wide spread variation in dosing strategies while using BSA based on individual physicians or institution practice. For example the choice between using the actual, ideal or adjusted body weight to calculate the dose might result in significant differences in doses administered [8-10]. This explains the acceptance of dose rounding within certain limits.

The purposes of this study are to determine the theoretical cost saving related to the dose rounding process of biological agents and chemotherapy agents within $15 \%$ and $10 \%$ respectively and to assess the adult oncologist's opinion about the consensus of dose rounding.
Corresponding author Nagwa Ibrahim, Pharm D, FAlHQ Clinical Pharmacist Specialist Oncology/ Hematology PSMMC Adjunct Assistant Professor, Pharmacy School, King Saud University Riyadh, Saudi Arabia nag_ibrahim@hotmail.com

\section{Disclosure}

This research received no specific grant from any funding agency in the public, commercial, or not-for-profit sectors 


\section{MATERIALS AND METHODS}

Data obtained prospectively during April 2011 from anticancer prescribing database in Department of Pharmaceutical Services at Prince Sultan Military Medical City (PSMMC), Saudi Arabia. Data includes the entered patient's body surface area (height and weight), the prescribed chemotherapy regimen and the doses administered. Data were identified and collected for all chemotherapy and targeted therapy orders prescribed by adult oncologists either from outpatient clinics or inpatient wards. Prescriptions include all types of cancer for patients in curative settings, metastatic and palliative care. We identified prescriptions in doses that might be rounded to an amount within $15 \%$ for biological therapy and up to $10 \%$ for chemothe-

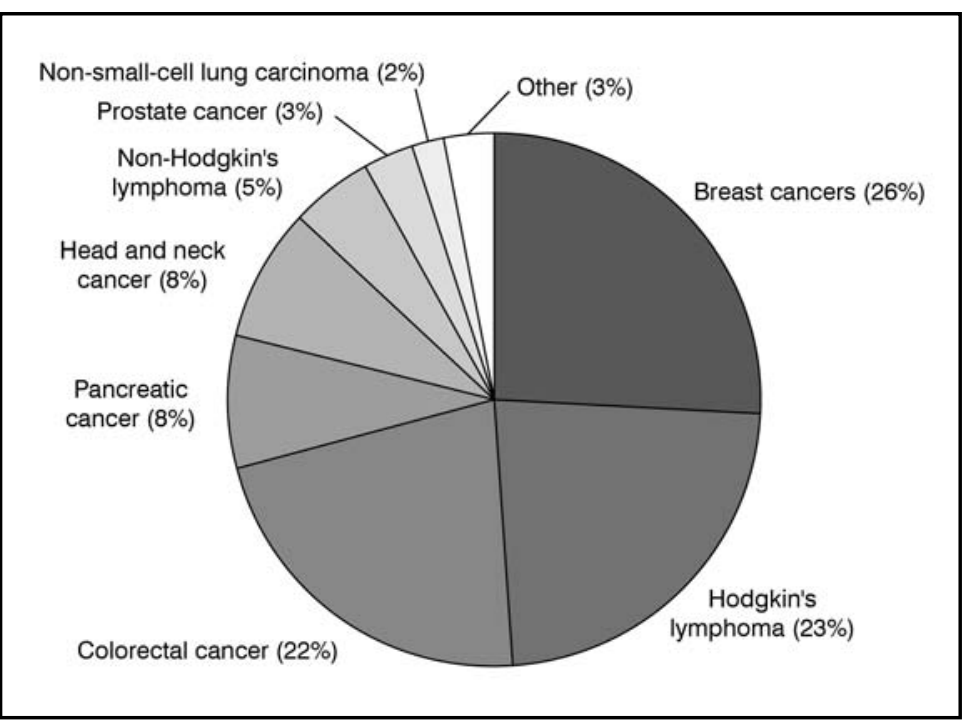

Figure 1. Percentage of prescriptions depending on type of cancer

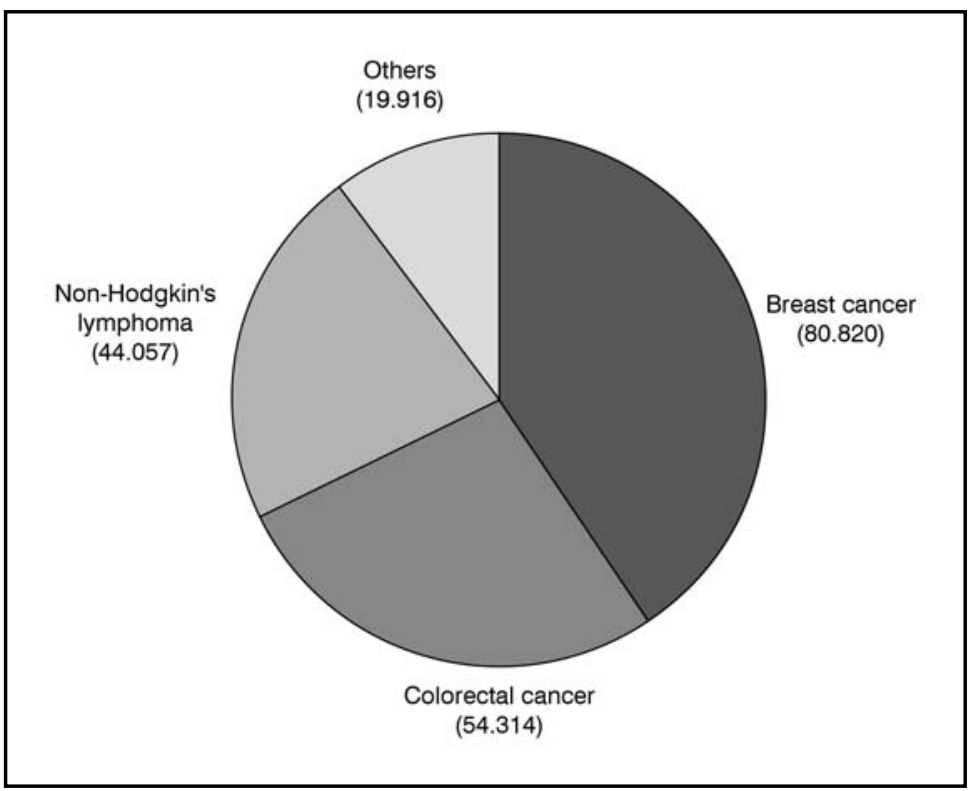

Figure 2. Cost saving (USD) per year depending on type of cancer rapy drugs. The difference between the actual given dose and the presumed rounded dose has been used to calculate the cost saving. Doses which are within the standard vial size (full dose given) were not included in the analysis. Specific drug costs were provided by the purchasing department. An approval from Ethical and Research Committee was achieved. A survey was distributed to all adult oncologists in PSMMC to seek their acceptability for dose rounding within the identified percentage for dose deviation.

\section{RESULTS}

We collected two hundred and thirty three oncology prescriptions including chemotherapy and targeted therapy. These orders were processed by the adult oncology satellite pharmacy during the period of data collection. Ninety six orders (41\%) out of 233 prescriptions fulfilled the rounding criteria. We performed a theoretical analysis for example a $108 \mathrm{mg}$ dose would be rounded to $100 \mathrm{mg}$ (7.4\% dose deviation). Data was extrapolated from the determined monthly cost savings. The potential cost savings from dose rounding per year was $\$ 192,800$. The highest cost saving was for breast cancer orders. It was around $\$ 80,820(42 \%)$, followed by colorectal cancer $\$ 47,965(25 \%)$, while in nonHodgkin's lymphoma cost savings was \$ $45,107(23 \%)$ and for other types of cancer that include non small cell lung cancer, prostate and ovarian cancer, in addition to head and neck cost savings was $\$ 18,867(10 \%)$ (Figures 1,2).

We surveyed the oncologists we have in adult oncology department. We received 10 responses $(100 \%)$. All of them accepted dose rounding up to $10 \%$ in both biologic and chemotherapy drugs.

\section{DISCUSSION}

Drug waste is defined as the consequence of an inappropriate disposal of unused or partially used drug ampoules, vials, or syringes [8]. Inefficiency of drug use and waste production may lead to a distinct economic loss, though experiences are limited and most studies are focused on other therapeutic areas $[8,11]$.

Pharmacy departments have looked to minimize cost through oncology medications preparation procedure. Few studies about cost minimization through dose rounding were published. Some of them were applied to the nearest vial available mainly for targeted therapy such as rituximab which is available as $400 \mathrm{mg}$ and $100 \mathrm{mg}$ vials. It is approved by 
FDA for non-Hodgkin's lymphoma (NHL). If the dose calculated is $750 \mathrm{mg}$ it could be rounded to $700 \mathrm{mg}$ by $6.7 \%$ dose deviation. In such a case one vial of $400 \mathrm{mg}$ plus 3 vials of $100 \mathrm{mg}$ or 7 vials of $100 \mathrm{mg}$ will be used. Accordingly we will save one $100 \mathrm{mg}$ vial [5-7]. Another study applied dose rounded to chemotherapy such as oxaliplatin which is used heavily in colorectal cancer protocols. It is available as $100 \mathrm{mg}$ and $50 \mathrm{mg}$ vials. If the dose calculated is $163 \mathrm{mg}$ it could be rounded to $150 \mathrm{mg}$ by $8 \%$ dose deviation. Accordingly we can save one $50 \mathrm{mg}$ vial. It is the same with other chemotherapy drugs as gemcitabine, docetaxel and targeted therapy as cetuximab and bevacizumab [4]. Reported cost avoidance is significant in these studies. However impact on clinical outcomes while applying dose rounding was not studied but recommended to be considered in future papers. Physician's opinion was surveyed as well by some authors and concluded that oncologists accepted dose rounding by up to $10 \%$ for chemotherapy and targeted therapy. We thought of conducting a pilot study by collecting data through April 2011 prescriptions from adult oncology satellite pharmacy. Data was extrapolated and cost avoidance calculated as annualized saving which is considered as study limitation. A single month's tally of orders may not provide a true picture of cancer types treated over a period of one year. It might not represent the cost avoidance over a year, it is just estimation.

We surveyed oncologist's opinion. They were questioned for the accepted percentage of dose deviation either in chemotherapy or targeted therapy. They agreed for up to $10 \%$ dose deviation in all treatment settings. Our study results are matching with other international studies. However we need to survey the oncologist's opinion in a larger scale at the region.

Based on the previous studies outcome including our study, dose rounding has a significant economic impact on cost saving and reduction of pharmaceutical department expenditures. It is crucial to keep in mind dose adjustment without affecting efficacy and safety of treatment. The size of problem in this field is little known. All studies including ours did not measure the clinical outcome due to small number of enrolled patients which is considered as study limitation. Impact of dose rounding to patient's clinical outcomes is warranted to be considered in future studies.

Some other questions need to be considered, will dose rounding initiated by clinician or pharmacists? In case of implementation should we consider dose adjustment based on a standard institutional policy? Further research to answer these questions is essential.

In addition, I suggest other approaches need to be considered for cost saving such as the use of multi-dose vials, utilization of the suitable vial size based on the convenience and unit pricing, and development of internal policy for implementation, periodic follow up and evaluation of policy implementation.

\section{CONCLUSION}

Our experience confirms the feasibility of dose rounding of chemotherapy and biologic drugs to an amount up to $10 \%$. Application will lead to significant cost savings and reduction in pharmacy department expenditures.

\section{REFERENCES}

1. Sullivan R, Peppercorn J, Sikora K, et al. Delivering affordable cancer care in high-income countries. Lancet Oncol 2011; 12: 933-80; http://dx.doi.org/10.1016/S1470-2045(11)70141-3

2. Jarkowski A 3rd, Nestico JS, Vona KL, et al. Dose rounding of ipilimumab in adult metastatic melanoma patients results in significant cost saving. J Oncol Pharm Practice 2013 [Epub ahead of print]; http://dx.doi. org/10.1177/1078155213476723

3. Fasola G, Aita M, Marini L, et al; Drug waste minimization and cost-containment in medical oncology: Two year results of a feasibility study. BMC Health Serv Res 2008; 8: 70; http://dx.doi.org/10.1186/1472-6963-8-70

4. Field K, Zelenko A, Kosmider S, et al. Dose rounding of chemotherapy in colorectal cancer: an analysis of clinician attitudes and the potential impact on treatment costs. Asia Pac J Clin Oncol 2010; 6: 203-9; http://dx.doi. org/10.1111/j.1743-7563.2010.01297.x

5. Dooley MJ, Singh S, Michael M. Implications of dose rounding of chemotherapy to the nearest vial size. Support Care Cancer 2004; 12: 653-6

6. Winger BJ, Clements EA, DeYoung JL, et al. Cost saving from dose rounding of biologic anticancer agents in adults. J Oncol Pharm Pract 2011; 17: 246-51; http://dx.doi.org/10.1177/1078155210366171

7. Patel S, Le A. Rounding rituximab dose to nearest vial size. J Oncol Pharm Pract 2013; 19: 218-21; http://dx.doi. org/10.1177/1078155212462439

8. Gurney H. Dose calculation of anticancer drugs: a review of the current practice and introduction of an alternative. J Clin Oncol 1996; 14: 2590-611 
9. Sawyer M, Ratain MJ. Body surface area as a determinant of pharmacokinetics and drug dosing. Invest New Drugs 2001; 19: 171-7

10. Nava-Ocampo AA, Alarcón-Almanza JM, Moyao-García D, et al. Undocumented drug utilization and drug waste increase costs of pediatric anesthesia care. Fundam Clin Pharmacol 2004; 18: 107-12

11. Diehl LD, Goo ED, Sumiye L, et al. Reducing waste of intravenous solutions. Am J Hosp Pharm 1992; 49: 106-8

12. Favier M, Fliche E, Bressolle F. Economic benefit of a centralized reconstitution unit of cytotoxic drugs in isolator. J Oncol Pharm Pract 1996; 2: 182-5; http://dx.doi.org/10.1177/107815529600200308

13. Gillerman RG, Browning RA. Drug use inefficiency: a hidden source of wasted health care dollars. Anesth Analg 2000; 91: 921-4 